\title{
APLICACIÓN DE VOLTAMPEROMETRÍA DE REDISOLUCIÓN ANÓDICA PARA LA DETERMINACIÓN DE ANTIMONIO
}

\author{
Hugo Ítalo Romero-Bonilla $\mathrm{a}^{\mathrm{*}^{*}}$, Thalía Gabriela Vera-Infante ${ }^{\mathrm{a}}$, \\ Felipe Fernando Redrovan-Pesantez ${ }^{\mathrm{b}}$, Jennifer del Cisne Jumbo-Sari ${ }^{\mathrm{a}}$, \\ Freddis Franco Pesantez
}

\begin{abstract}
RESUMEN
Se validó un método voltamétrico para determinar la concentración de antimonio en medio acuoso. Se usó la técnica voltamperométrica de redisolución anódica, utilizando un potenciostato. Se aplicó cronoamperometría a $\mathrm{E}=-1,2 \mathrm{~V}$ durante $50 \mathrm{~s}$. Se aplicó una voltametría de barrido lineal con potenciales entre $\mathrm{E}_{\mathrm{i}}=-1,2 \mathrm{~V}$ y $\mathrm{E}_{\mathrm{f}}=0,5 \mathrm{~V}$ a una velocidad de $20 \mathrm{mV} / \mathrm{s}$. Esto se llevó a cabo en solución electrolítica $\mathrm{H}_{3} \mathrm{PO}_{4} 0,5 \mathrm{M}$, donde se puede observar un incremento en el pico de intensidad de corriente en función de la concentración de las soluciones evaluadas a un potencial de $84,96 \mathrm{mV}$. Para validar el método se determinó la linealidad, precisión, límite de detección y límite de cuantificación. Se prepararon cuatro concentraciones de antimonio por triplicado, en un rango de 1, 5, 10, 15, $20 \mathrm{mg} / \mathrm{L}$, respectivamente. Se construyó una curva de calibración de concentración experimental expresada como intensidad de corriente (I) vs concentración teórica. La linealidad fue demostrada partiendo del coeficiente de proporcionalidad de 0,9979 con coeficiente de variación $(\mathrm{CV})$ de 18,2\%. El límite de detección y cuantificación tuvieron valores de 1,041 $\mathrm{mg} / \mathrm{L}$ y $1,718 \mathrm{mg} / \mathrm{L}$, respectivamente.
\end{abstract}

Palabras clave: validación, electroanalítica, voltamperometría, antimonio.

\section{APPLICATION OF ANODIC REDISOLUTION VOLTAMPEROMETRY FOR THE DETERMINATION OF ANTIMONIUM}

\begin{abstract}
A voltammetric method was validated to determine the concentration of antimony in aqueous medium. The voltammetric technique of anodic redissolution was used, using a potentiostat. Chronoamperometry was applied at $\mathrm{E}=-1,2 \mathrm{~V}$ for $50 \mathrm{~s}$. A linear sweep voltammetry was applied with potentials between $E_{i}=-1,2 \mathrm{~V}$ and $E_{f}=0,5 \mathrm{~V}$ at a speed of $20 \mathrm{mV} / \mathrm{s}$. This was

a Grupo de Investigación de Aplicaciones Electroanalíticas, Laboratorio de Electroanálisis y Bioenergía, Universidad Técnica de Machala, Ecuador.

${ }^{\text {b }}$ Facultad de Ciencias Químicas y de la Salud, Universidad Técnica de Machala, carrera de Ingeniería en Alimentos, Machala, Ecuador

*aahromero@utmachala.edu.ec
\end{abstract}


carried out in $0,5 \mathrm{M} \mathrm{H}_{3} \mathrm{PO}_{4}$ electrolytic solution, where an increase in the current intensity peak can be observed as a function of the concentration of the solutions evaluated at a potential of $84,96 \mathrm{mV}$. To validate the method, linearity, precision, limit of detection and limit of quantification were determined. Four concentrations of antimony were prepared in triplicate, in a range of $1,5,10,15,20 \mathrm{mg} / \mathrm{L}$, respectively. A calibration curve of experimental concentration expressed as current intensity (I) vs theoretical concentration was constructed. The linearity was demonstrated starting from the coefficient of proportionality of 0,9979 with coefficient of variation (CV) of $18,2 \%$. The limit of detection and quantification had values of $1,041 \mathrm{mg} / \mathrm{L}$ and $1,718 \mathrm{mg} / \mathrm{L}$ respectively.

Key words: validation, electroanalytical, voltamperometry, antimony.

\section{INTRODUCCIÓN}

La contaminación en el medio ambiente podría causar daños. A fin de reducir la contaminación ambiental y mitigar la degradación resultante del suelo y de los recursos hídricos, se deben determinar las concentraciones de metales pesados ${ }^{1}$. Es importante desarrollar métodos analíticos altamente sensibles para evaluar contaminantes en el suelo, el agua y el aire para mejorar la calidad del ambiente y la vida humana ${ }^{2}$.

Las implicaciones ambientales y los efectos adversos sobre los organismos vivos se deben principalmente a la contaminación por metales ${ }^{3}$. Estas investigaciones documentadas han conducido al desarrollo de una serie de directrices, políticas y leyes ambientales para varios de estos elementos, tales como: $\mathrm{As}, \mathrm{Cd}, \mathrm{Sb}, \mathrm{Cr}, \mathrm{Cu}, \mathrm{Hg}$, Pb, otros. Sin embargo, existen otros elementos traza, donde todavía hay una brecha en nuestro conocimiento sobre su especiación química, así como su potencial ecotoxicológico ${ }^{4}$.

Actualmente, los métodos más comunes para cuantificar metales son relativamente complejos y abarcan un pretratamiento que lleva mucho tiempo para lograr su separación. Esto resulta bastante costoso $0^{5}$ e incluye otros procesos como filtración, centrifugación, vacío o ultrafiltración centrífuga, diálisis y cromatografía líquida de alto rendimiento ${ }^{6}$.

Una de las ventajas de utilizar métodos instrumentales de análisis es que son capaces de detectar y determinar cantidades de analito mucho más pequeñas que los métodos clásicos?

Uno de los principales problemas que se presentan cuando se trabaja con concentraciones a nivel de trazas se da a la hora de informar sobre la presencia o ausencia de un analito determinado. En este sentido, es importante llevar a cabo la determinación tanto del límite de detección como del límite de cuantificación ${ }^{8}$.

En los últimos años ha surgido la necesidad de desarrollar nuevas tecnologías y métodos para la seguridad y el control de calidad en una gama de diferentes aplicaciones ${ }^{9}$. 
Los metales pesados son elementos potencialmente tóxicos, cuya presencia en el medio ambiente se ha incrementado notablemente en las últimas décadas, fundamentalmente por la acción del hombre. La contaminación metálica supone una amenaza medioambiental importante para los seres vivos ${ }^{10}$. La existencia de Sb en el ambiente es una consecuencia de la utilización de materias primas que contienen este elemento en la fabricación de vidrio, la cerámica, frenos de automóviles, retardantes de llama, etc. ${ }^{11}$

La exposición a cantidades relativamente altas de antimonio durante un largo periodo de tiempo puede provocar irritación de los ojos, piel y pulmones. Si la exposición continúa se pueden producir efectos más graves, tales como enfermedades pulmonares, problemas de corazón, diarrea, vómitos severos y úlceras estomacales. Estos efectos sobre la salud nos han hecho estar más alerta acerca de los peligros de la exposición al antimonio ${ }^{12}$.

La utilización de técnicas electroanalíticas depende de la superficie del electrodo, que debe proporcionar una correlación señal-ruido y además buena reproducibilidad. Para la manipulación de esta técnica se debe aplicar un potencial a un electrodo, y también se debe hacer un seguimiento de la corriente (I) resultante, que se lo realiza en una celda electroquímica en medio acuoso ${ }^{13}$.

La electroquímica es una opción accesible e innovadora para la cuantificación de trazas de metales pesados, se basa en el comportamiento de la corriente y el potencial sobre la superficie del electrodo de trabajo. Asimismo, la voltamperometría constituye una alternativa de amplio espectro, económica y amigable con el medio ambiente.

La detección de iones trazas de metales pesados como el cobre, el plomo y el antimonio es trascendental en el monitoreo de la calidad del medio ambiente y la salud ${ }^{14}$. La voltamperometría de redisolución anódica (ARV) es una potente técnica analítica para la detección de metales traza por su alta sensibilidad y reproducibilidad ${ }^{15}$.

El análisis de separación electroquímico es reconocido como una de las técnicas más convenientes para la medición de trazas de iones de metales pesados, en particular, debido a su capacidad de pre-concentrar analitos en la superficie del electrodo de trabajo, lo que permite la cuantificación de iones de metales pesados a un nivel bajo microgramos por litro o incluso nanogramos por litro niveles de concentración ${ }^{16}$

La voltamperometría de redisolución anódica pre-concentra el analito mediante su reducción en una película delgada sobre el electrodo de trabajo. Adicionalmente, el potencial se vuelve más positivo y oxida la especie en la solución. La corriente medida durante la oxidación es proporcional a la cantidad de analito que fue depositada ${ }^{17}$.

Las ventajas analíticas de las técnicas voltamperométricas incluyen una excelente sensibilidad con amplio rango de concentración lineal útil tanto para especies inorgánicas como orgánicas (1/1012 a 1/10 M), gran cantidad de disolventes y electrolitos útiles, un amplio rango de 
temperaturas, tiempos de análisis rápidos (segundos), determinación simultánea de varios analitos, la capacidad de determinar cinética y parámetros mecánicos ${ }^{18}$.

La validación de un método analítico es el proceso establecido por los estudios de laboratorio para garantizar el cumplimiento de una serie de requisitos indispensables para la futura aplicación analítica del método. Es necesaria porque proporciona un alto grado de confianza y seguridad del proceso productivo, o del método analítico, así como también en la calidad de los resultados. Estos requisitos son expresados en términos de parámetros analíticos ${ }^{19}$. El objetivo final de la validación del método de análisis es asegurar que cada medición futura en el análisis de rutina será lo suficientemente cerca del valor verdadero desconocido para el contenido del analito en la muestra ${ }^{20}$.

Sin embargo, las técnicas electroquímicas como la voltamperometría ofrecen una alta sensibilidad, mayor precisión y menores costos de operación ${ }^{21}$.

El objetivo de la presente investigación fue desarrollar un método electroanalítico preciso, selectivo y lineal para determinar antimonio mediante una técnica sencilla, de bajo costo y amigable con el medio ambiente como es la voltamperometría de redisolución anódica.

\section{PARTE EXPERIMENTAL}

Para la obtención de datos se utilizó un potenciostato PARSTAT multicanal marca Princeton Applied Research con software Versa Studio Potenciostat. Para llevar a cabo el registro de los voltamperogramas, se contó con un sistema de tres electrodos, tales como: carbón vítreo como electrodo de trabajo, electrodo de $\mathrm{Ag} / \mathrm{AgCl}$ como electrodo de referencia y un electrodo de platino como contraelectrodo.

Toda la cristalería utilizada en el desarrollo del método voltamperométrico se lavó con un detergente libre de metales, luego con agua destilada y posteriormente se colocó en baños de ácido nítrico $1 \mathrm{M}$, por una hora, finalmente se enjuagó con agua desionizada.

Se preparó un electrolito soporte a partir de ácido fosfórico $\left(\mathrm{H}_{3} \mathrm{PO}_{4}\right)$ 0,5 $\mathrm{M}$; grado analítico marca SIGMA-ALDRICH. Además, se necesitó de un estándar de antimonio ( $\mathrm{Sb}$ ) de 1000 $\mathrm{mg} / \mathrm{L}$ marca AccuStandard, a partir de la cual se prepararon diluciones de 1, 5, 10, 15 y 20 $\mathrm{mg} / \mathrm{L}$ de $\mathrm{Sb}$ en electrolito soporte, para realizar la validación del método. Adicionalmente, se burbujeó nitrógeno al $99 \%$ de pureza para eliminar el oxígeno en el sistema acuoso.

Por su parte, la limpieza del electrodo de trabajo de carbón vítreo se realizó con una solución de alúmina de 0,3 y 0,1 micrones. Para asegurar la limpieza de la celda electrolítica, se empleó lavados con ácido nítrico $1 \mathrm{M}$, por una hora. Por otro lado, se realizó la activación electroquímica del electrodo de carbón vítreo para obtener una superficie limpia mediante períodos de voltamperometría de 50 ciclos en solución de H3PO4 0,5 M con un barrido de potencial $\mathrm{E}_{\mathrm{i}}=-1 \mathrm{~V} \mathrm{y}_{\mathrm{f}}=1 \mathrm{~V}$ a una velocidad de barrido $(v)=20 \mathrm{mV} / \mathrm{s}$ 


\section{Método electroanalítico}

La voltamperometría de redisolución anódica fue la técnica utilizada en este trabajo, para lo cual se realizó en primer lugar una cronoamperometría a $\mathrm{E}=-1,2 \mathrm{~V}$ durante un tiempo de pre-concentración de 50 obteniendo la electrodeposición del antimonio metálico $\left(\mathrm{Sb}^{0}\right)$ en la superficie del electrodo de carbón vítreo. Seguidamente, se aplicó una voltametría de barrido lineal con potencial inicial $\left(\mathrm{E}_{\mathrm{i}}\right)$ de $-1,2 \mathrm{~V}$ y un potencial final $\left(\mathrm{E}_{\mathrm{f}}\right)$ de $0,5 \mathrm{Va}$ una velocidad de $20 \mathrm{mV} / \mathrm{s}$. El antimonio se oxidó desprendiéndose del electrodo de carbón vítreo a un potencial de $84,96 \mathrm{mV}$, lo cual generó un incremento de corriente que se observó en los voltamperogramas. Los análisis fueron realizados a temperatura ambiente y a presión atmosférica.

\section{Validación del método analítico}

Con el objetivo de validar el método para la cuantificación de antimonio, se procedió con la determinación de linealidad, precisión y exactitud según lo reportado en la literatura ${ }^{17}$.

\section{Linealidad del método}

Se prepararon cuatro concentraciones de antimonio por triplicado, en un rango de $1,5,10$, $15,20 \mathrm{mg} / \mathrm{L}$, respectivamente. Se construyó una curva de calibración de concentración experimental expresada como intensidad de corriente (I) vs concentración teórica. Los resultados se procesaron estadísticamente a través de Excel y se determinó: $\mathrm{r}$ (coeficiente de correlación lineal), $\mathrm{r}^{2}$ (coeficiente de determinación), a (intercepto) y b (pendiente).

Se realizó una evaluación de curva de calibración global (construida con más de una curva de calibración de las mismas características) en la cual se realizó una evaluación estadística de prueba t-Student, como un mejor indicador del modelo lineal.

Se determinó un valor de $\mathrm{t}$ con $\mathrm{n}-2$ grados de libertad y se comparó con el valor tabulado de t para el nivel de confianza requerido $(\alpha=0,05)$.

\section{Precisión}

Se analizaron 10 muestras para 5 niveles de concentración, a partir de los cuales se determinó la desviación estándar relativa. Según la Food ang Drug Administration - FDA (2001), la desviación estándar relativa (RSD) debe ser menor de $20 \%$ para concentraciones de nivel bajo, y menor de $15 \%$ para las concentraciones medias y altas.

\section{Límite de detección}

El límite de detección de un método analítico es la mínima cantidad de analito presente en la muestra que se puede detectar, aunque no cuantificar, a través de las condiciones experimentales descritas para dicho método de acuerdo a la siguiente fórmula: 


$$
L O D=\frac{3 \sigma_{B}}{b}
$$

Donde:

$\sigma_{B}=$ Desviación estándar

$\mathrm{b}=$ Pendiente de la curva de calibración

\section{Límite de cuantificación}

El límite de cuantificación de un método analítico es la mínima cantidad de analito presente en la muestra que se puede cuantificar bajo las condiciones experimentales descritas para dicho método, para lo cual se utilizó la siguiente fórmula:

$$
L O Q=\frac{10 \sigma_{B}}{b}
$$

Donde:

$\sigma_{B}=$ Desviación estándar

$\mathrm{b}=$ Pendiente de la curva de calibración

\section{RESULTADOS Y DISCUSIÓN}

En la figura 1 se muestra la cronoamperometría realizada para la electrodeposición del antimonio sobre el electrodo de carbón vítreo (electrodo de trabajo) en forma de $\mathrm{Sb}$ metálico a partir de una solución 1 ppm de antimonio, utilizando como electrólito $\mathrm{H}_{3} \mathrm{PO}_{4} 0,5 \mathrm{M}$.

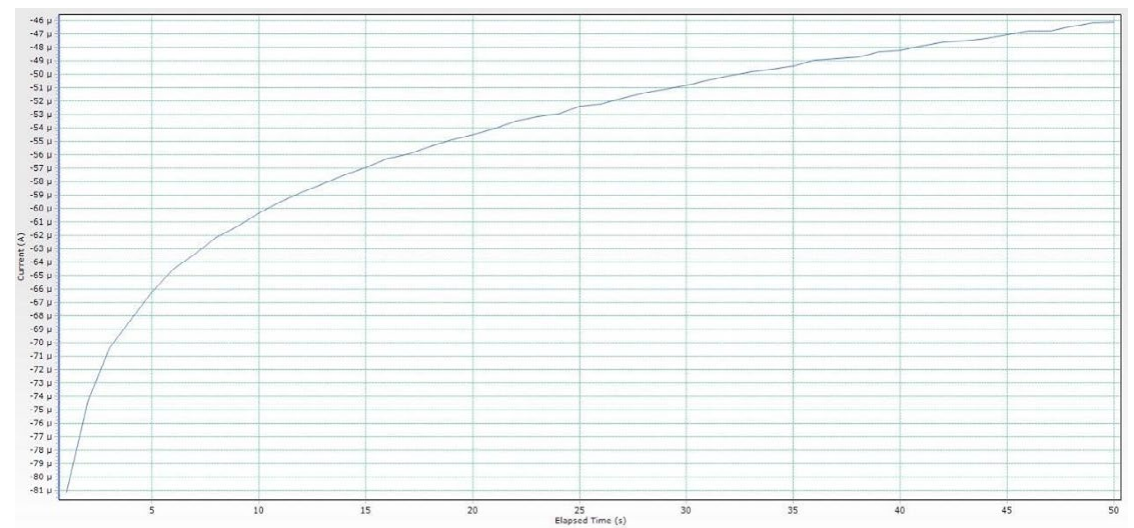

Figura 1. Cronoamperograma del $\mathrm{Sb}$ en solución de $1 \mathrm{mg} / \mathrm{L} \mathrm{Sb}$ y $0,5 \mathrm{~mol} / \mathrm{L} \mathrm{H}_{3} \mathrm{PO}_{4}$, cátodo de carbón vítreo. $\mathrm{E}=-1,2 \mathrm{~V}$ y $\mathrm{t}=50 \mathrm{~s}$. 
Luego de la electrodeposición mostrada en la figura 1, se procedió con la etapa de redisolución el antimonio mediante voltamperometría de barrido lineal (figura 2), donde este metal se oxidó desprendiéndose del electrodo de carbón vítreo a un potencial de $84,96 \mathrm{mV}$, lo que generó un incremento de corriente. Este fenómeno nos permitió cuantificar por regresión lineal de una curva de calibración la concentración en solución de los iones oxidados. A partir de la figura 2, la altura del pico (corriente máxima), o en su defecto, el área del pico (corriente total) pueden ser usadas para calcular la concentración del analito ( $\mathrm{Sb}$ ) en la solución. La figura 2 muestra las propiedades del voltamperograma obtenido.

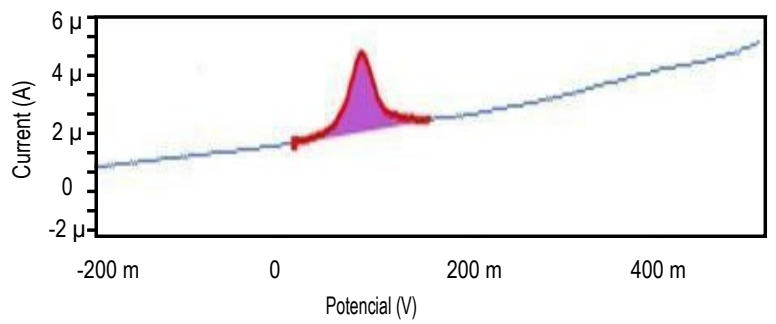

$$
\begin{gathered}
\text { Peak Analysis: } \\
\text { Peak I: } \\
4.612 \mu \mathrm{A} \\
\text { Peak E: } \\
84.957 \mathrm{mV} \\
\text { FWHM: } \\
31.87 \mathrm{~V} \\
\text { Area: } \\
1.626 \mu \mathrm{C} \\
\text { Range: } \\
\text { 15mV to } 156.4 \mathrm{mV}
\end{gathered}
$$

Figura 2. Voltamperograma de $\mathrm{Sb}$ en solución de $1 \mathrm{mg} / \mathrm{L}$ Sb en $0,5 \mathrm{~mol} / \mathrm{L} \mathrm{H}_{3} \mathrm{PO}_{4}$, cátodo de carbón vítreo, corriente $(\mathrm{I})=4,612 \mu \mathrm{A} ; \mathrm{v}=20 \mathrm{mV} / \mathrm{s}, \mathrm{Ei}=-1,2 \mathrm{~V}, \mathrm{Ef}=0,5 \mathrm{~V}$; Eoxidación= 84,96 mV.

Por otro lado, la figura 3 muestra un voltamperograma de $\mathrm{Sb}$ en solución electrolítica $\mathrm{H}_{3} \mathrm{PO}_{4}$ 0,5 M, donde se puede observar cómo aumenta el pico de intensidad de corriente en función de la concentración de las soluciones evaluadas a un potencial de $84,96 \mathrm{mV}$.

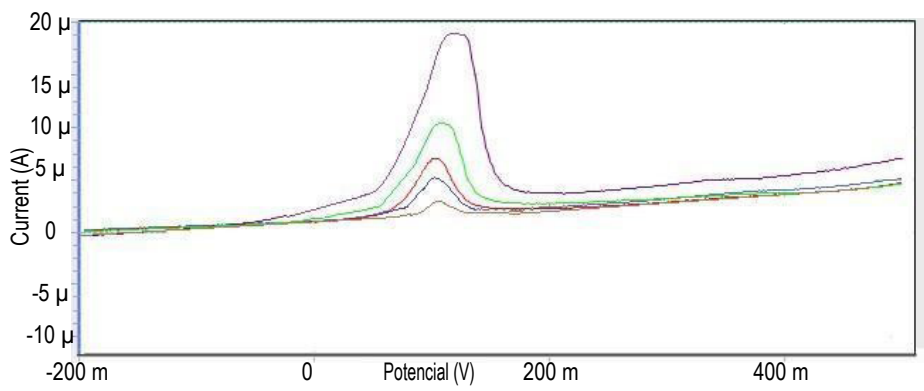

Figura 3. Voltamperograma de $\mathrm{Sb}$ en solución de $1 \mathrm{mg} / \mathrm{L} \mathrm{Sb}$ (línea azul), $10 \mathrm{mg} / \mathrm{L} \mathrm{Sb}$ (línea roja), $15 \mathrm{mg} / \mathrm{L} \mathrm{Sb}$ (línea verde) y $20 \mathrm{mg} / \mathrm{L} \mathrm{Sb}$ (línea lila) en $0,5 \mathrm{~mol} / \mathrm{L} \mathrm{H}_{3} \mathrm{PO}_{4}$, cátodo de carbón vítreo; $\mathrm{v}$ $=20 \mathrm{mV} / \mathrm{s} ; \mathrm{Ei}=-1,2 \mathrm{~V} ; \mathrm{Ef}=0,5 \mathrm{~V} ;$ Eoxi $=84,957 \mathrm{mV}$.

Los resultados experimentales de la linealidad del sistema se muestran en la figura 4 y los resultados del procesamiento estadístico se resumen en la tabla 1. Según el cumplimiento de los parámetros estadísticos establecidos (tabla 1) se valida la linealidad del sistema. El conjunto de estos resultados permitió afirmar que el método fue lineal en el rango estudiado, ya que se obtuvo una elevada proporcionalidad entre la respuesta obtenida y la concentración del analito, tal como se muestra en la figura 4 y se demuestra por el cumplimiento de todos los criterios estadísticos que derivan del análisis de la regresión para un nivel de confianza de $95 \%$. 
Estos resultados son similares a los obtenidos por Engin y $\mathrm{col}^{21}$, donde se obtuvo una ecuación de regresión lineal del pico de corriente $\left(\mathrm{I}_{\mathrm{p}}\right) ; \mathrm{I}_{\mathrm{p}}(\mu \mathrm{A})=2,20 \times 10^{4}(\mathrm{~mol} / \mathrm{L})-0,0024$; con un coeficiente de correlación, $\mathrm{r}^{2}=0,9989$; para $\mathrm{n}=5$ mediciones repetidas y con desviaciones

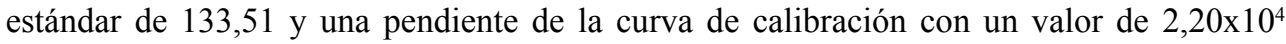
$\mu \mathrm{A} / \mathrm{M}$.

Tabla 1. Resultados del parámetro de linealidad para un nivel de confianza de $95 \%$.

\begin{tabular}{lll}
\hline Parámetro & Resultados & Criterio de aceptación \\
\hline & $\mathrm{Y}=0,745 \mathrm{x}+0,5019$ & $\mathrm{Y}=\mathrm{bx}+\mathrm{a}$ \\
0,9989 & $\mathrm{r} \geq 0,99$ \\
& 0,9979 & $\mathrm{r}^{2} \geq 0,99$ \\
Linealidad & $\operatorname{tcri}<\operatorname{tr}(\alpha=0,05 ; \mathrm{n}=5)$ & Intercepto no significativo \\
& & $\mathrm{tcri}<\operatorname{tr}(4,303)$ \\
& 0,745 & $\mathrm{~b} \cong 1$ \\
$\mathrm{t}=361,93$ & $\mathrm{t}$ alta
\end{tabular}

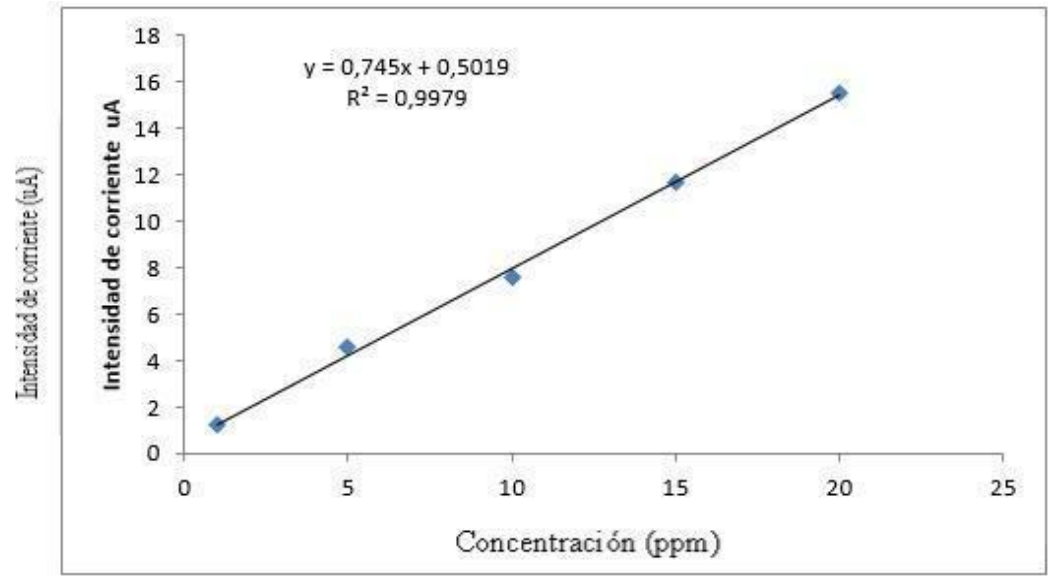

Figura 4. Curva de calibración para la determinación de Sb con adición de solución estándar (1, $5,10,15$ y $20 \mathrm{mg} / \mathrm{L})$. 
El coeficiente de variación (CV) obtenido a partir de los factores de respuesta es de 18,2\%, cual indica que el método es preciso, como se muestra en la tabla 2.

Tabla 2. Análisis de repetitividad del método.

\begin{tabular}{|c|c|c|}
\hline$X(\mu g / m L)$ & $\mathrm{Y}(\mu \mathrm{A})$ & $\mathbf{f}=\mathbf{Y} / \mathbf{X}$ \\
\hline 1 & 0,998 & 0,998 \\
\hline 1 & 1,198 & 1,198 \\
\hline 1 & 1,082 & 1,082 \\
\hline 1 & 1,009 & 1,009 \\
\hline 1 & 1,159 & 1,159 \\
\hline 1 & 0,951 & 0,951 \\
\hline 1 & 1,199 & 1,199 \\
\hline 1 & 1,116 & 1,116 \\
\hline 1 & 1,018 & 1,018 \\
\hline 5 & 4,548 & 0,909 \\
\hline 5 & 4,612 & 0,922 \\
\hline 5 & 4,855 & 0,971 \\
\hline 5 & 6,221 & 1,244 \\
\hline 5 & 5,694 & 1,138 \\
\hline 5 & 5,339 & 1,067 \\
\hline 5 & 5,268 & 1,053 \\
\hline 5 & 5,166 & 1,033 \\
\hline 5 & 5,723 & 1,144 \\
\hline 10 & 8,825 & 0,882 \\
\hline 10 & 9,385 & 0,938 \\
\hline 10 & 6,979 & 0,697 \\
\hline 10 & 7,492 & 0,749 \\
\hline 10 & 6,733 & 0,673 \\
\hline 10 & 7,558 & 0,755 \\
\hline 10 & 7,050 & 0,705 \\
\hline 10 & 6,150 & 0,615 \\
\hline 10 & 7,473 & 0,747 \\
\hline
\end{tabular}


Tabla 2. Análisis de repetitividad del método. Continuación

\begin{tabular}{|c|c|c|}
\hline 15 & 10,831 & 0,722 \\
\hline 15 & 11,614 & 0,774 \\
\hline 15 & 11,094 & 0,739 \\
\hline 15 & 11,590 & 0,772 \\
\hline 15 & 10,736 & 0,715 \\
\hline 15 & 11,689 & 0,779 \\
\hline 15 & 11,584 & 0,772 \\
\hline 15 & 11,399 & 0,759 \\
\hline 15 & 10,223 & 0,681 \\
\hline 20 & 16,816 & 0,840 \\
\hline 20 & 16,294 & 0,814 \\
\hline 20 & 18,245 & 0,912 \\
\hline 20 & 17,357 & 0,867 \\
\hline 20 & 17,452 & 0,872 \\
\hline 20 & 18,047 & 0,902 \\
\hline 20 & 18,782 & 0,939 \\
\hline 20 & 18,639 & 0,932 \\
\hline 20 & 17,973 & 0,898 \\
\hline Promedio de $f$ & 0,904 & \\
\hline $\mathbf{s}$ & 0,164 & \\
\hline RSD & 0,182 & \\
\hline CV (\%) & 18,2 & \\
\hline
\end{tabular}

\section{CONCLUSIONES}

Se logró determinar que el potencial del pico de oxidación del antimonio en las condiciones estudiadas fue de $84,96 \mathrm{mV}$. Por su parte, la linealidad del método fue demostrada a partir del coeficiente de proporcionalidad de 0,99793 entre la respuesta obtenida y la concentración del analito. El coeficiente de variación (CV) obtenido tuvo un valor de 18,2 \%, lo cual indica que la precisión del método es aceptable. Adicionalmente, se encontró que el límite de detección y límite de cuantificación corresponden a valores de 1,041 mg/L y 1,718 mg/L, respectivamente. 


\section{AGRADECIMIENTO}

Los autores agradecen al Grupo y Semillero de Investigación Aplicaciones Electroanalíticas (AEA) de la Carrera de Ingeniería Química de la Universidad Técnica de Machala, Ecuador, por el apoyo brindado al desarrollo del presente trabajo de investigación.

\section{REFERENCIAS BIBLIOGRÁFICAS}

1. Lu Y, Liang X, Niyungeko C, Zhou J, Xu J, Tian G. A review of the identification and detection of heavy metal ions in the environment by voltammetry. Talanta. 2018; 178: 324-338.

2. Lee S, Bong S, Ha J, Kwak M, Park S-K, Piao Y. Electrochemical deposition of bismuth on activated graphene-nafion composite for anodic stripping voltammetric determination of trace heavy metals. Sens Actuators B Chem. 2015; 215:62-69.

3. Esteban M., Ariño C., Díaz JM, Serrano N., Dago A., Pérez C. Sensores voltamperométricos para la determinación de iones metálicos. Actualidad Analítica, 2016; 53: 15-1.

4. Cobelo-García A, Filella M. Electroanalytical techniques for the quantification of technology-critical elements in environmental samples. Curr Opin Electrochem. 2017; 1: 78-90.

5. Fang H, Zhang J, Zhou S, Dai W, Li C, Du D. Submonolayer deposition on glassy carbon electrode for anodic stripping voltammetry: An ultra sensitive method for antimony in tap water. Sens Actuators B Chem. 2015; 210: 113-119.

6. Romih T, Hočevar SB, Jemec A, Drobne D. Bismuth film electrode for anodic stripping voltammetric measurement of silver nanoparticle dissolution. Electrochim Acta. 2016; 188: 393-397.

7. Atachao ER. Determinación de los niveles de plomo y cadmio en labiales en barra multimarca mediante espectrofotometría de absorción atómica según comercialización en Lima cercado. [Tesis de grado]. Lima: Universidad Particular Inca Garcilazo de la Vega; 2017.

8. Renteria B, Zepeda F. Estudio prelimar [ie preliminar] de un método voltamperométrico para la determinación de plomo en agua potable. [Tesis de grado]. León: Universidad Nacional Autónoma de Nicaragua; 2008.

9. Gil Z, López V. Aplicación de las nuevas tecnologías de información y comunicación (NTIC) y las diferencias de su concepción en la banca pública y privada, en Venezuela. Barcelona, Venezuela: Universidad de Oriente; 2008.

10. Alonso J, García MA, Pérez López JM, Melgar MJ. Acumulación de metales pesados en macromicetos comestibles y factores que influyen en su captación. Rev Tox. 2004; 21(1): 11-15.

11. Ordoñes J, Fernández L, Romero H, Carrera P, Alvarado J. Electrochemical generation of antimony volatile species, stibine, using gold and silver mercury amalgamated cathodes and determination of $\mathrm{Sb}$ by flame atomic absorption spectrometry. Talanta. 2015; 141: 259-266. 
12. Romero H, Vera T, García C, Lapo B, Pesantez F. Desarrollo y validación de un método voltamétrico para la determinación de antimonio en medio acuoso. Conf Proc IEEE Eng Med Biol Soc. 2017; 1(1): 736-745.

13. Zhiñin G, Esthefania A, Molina Ramirez EE. Aplicación de técnicas electroanalísticas en la determinación de plomo en agua potable. Machala: Universidad Técnica de Machala; 2015.

14. Lin H, Li M, Mihailovič D. Simultaneous Determination of Copper, Lead, and Cadmium Ions at a Mo6S9-xIx Nanowires Modified Glassy Carbon Electrode Using Differential Pulse Anodic Stripping Voltammetry. Electrochim Acta. 2015; 154: 184-189.

15. Zhang M-R, Pan G-B. Porous GaN electrode for anodic stripping voltammetry of silver(I). Talanta . 2017; 165: 540-544.

16. Wang J. Analytical Electrochemistry. 3rd Ed. Hoboken, NJ, USA: John Wiley \& Sons; 2006.

17. Lledó D, Calixto F. Aplicación de métodos de preconcentración/determinación de metales pesados en sedimentos y aguas: membranas líquidas-espectroscopía atómica y voltametría de redisolución. [Tesis de grado]. Cádiz: Universidad de Cádiz; 2007.

18. Massanet López JA. Electrodes modification with silver nanoparticles for the detection of arsenic [Internet]. Barcelona: Universitat Politècnica de Catalunya; 2017. [Citado 18 de mayo 2019]. Disponible en: https://upcommons.upc.edu/bitstream/handle/2117/115476/ TFM.pdf?sequence $=1$ \&isAllowed $=\mathrm{y}$

19. Hidalgo C, Suárez Y, Fernández M. Validación de una técnica potenciométrica para determinar el grado de desacetilación de la quitosana. Ars Pharm. 2008; 49(3): 245-257.

20. González AG., Herrador MÁ. A practical guide to analytical method validation, including measurement uncertainty and accuracy profiles. Trends Analyt Chem. 2007; 26(3): 227238.

21. Engin C, Yilmaz S, Saglikoglu G, Yagmur S, Sadikoglu M. Electroanalytical investigation of paracetamol on glassy carbon electrode by voltammetry. Int J Electrochem Sci. 2015; 10: 1916-1925.

22. Ramírez C, Olarte E, Téllez E, Palma M. Validación del método analítico para la determinación de mercurio total en sangre humana por espectrofotometría de absorción atómica Zeeman RA-915+ con el módulo de pirólisis PYRO-915+. Rev Colomb Quím. 2013; 42(3): 11-16. 